\title{
QUEEN'S
UNIVERSITY
BELFAST
}

\section{Magnetic Characteristics of a High-layer-number NiFe/FeMn Multilayer}

Paterson, G. W., Goncalves, F. J. T., McFadzean, S., O'Reilly, S., Bowman, R., \& Stamps, R. L. (2015).

Magnetic Characteristics of a High-layer-number NiFe/FeMn Multilayer. Journal of Applied Physics, 118(20), [203903]. https://doi.org/10.1063/1.4936199

\section{Published in:}

Journal of Applied Physics

Document Version:

Publisher's PDF, also known as Version of record

Queen's University Belfast - Research Portal:

Link to publication record in Queen's University Belfast Research Portal

\section{Publisher rights}

Copyright (2015) AIP Publishing. This article may be downloaded for personal use only. Any other use requires prior permission of the author and AIP Publishing.

The following article appeared inPaterson, GW, Goncalves, FJT, McFadzean, S, O'Reilly, S, Bowman, R \& Stamps, RL 2015, 'Magnetic Characteristics of a High-layer-number NiFe/FeMn Multilayer' Journal of Applied Physics, vol 118, no. 20, 203903 and may be found at http://scitation.aip.org/content/aip/journal/jap/118/20/10.1063/1.4936199

\section{General rights}

Copyright for the publications made accessible via the Queen's University Belfast Research Portal is retained by the author(s) and / or other copyright owners and it is a condition of accessing these publications that users recognise and abide by the legal requirements associated with these rights.

\section{Take down policy}

The Research Portal is Queen's institutional repository that provides access to Queen's research output. Every effort has been made to ensure that content in the Research Portal does not infringe any person's rights, or applicable UK laws. If you discover content in the Research Portal that you believe breaches copyright or violates any law, please contact openaccess@qub.ac.uk. 


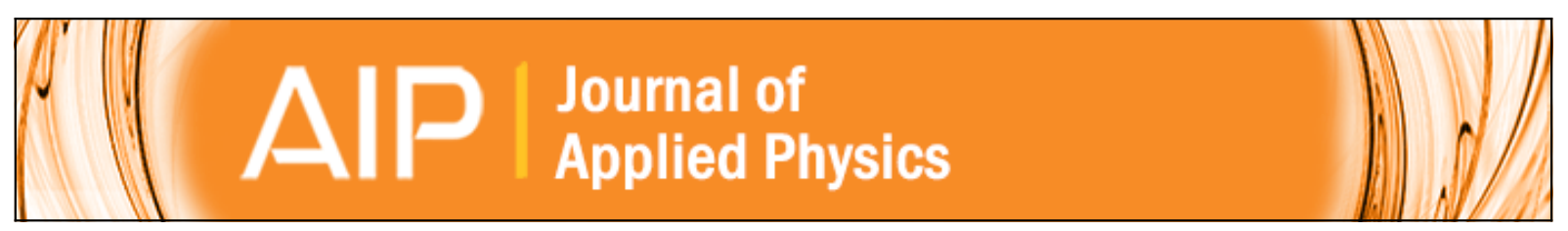

\section{Magnetic characteristics of a high-layer-number NiFe/FeMn multilayer}

G. W. Paterson, F. J. T. Gonçalves, S. McFadzean, S. O'Reilly, R. Bowman, and R. L. Stamps

Citation: Journal of Applied Physics 118, 203903 (2015); doi: 10.1063/1.4936199

View online: http://dx.doi.org/10.1063/1.4936199

View Table of Contents: http://scitation.aip.org/content/aip/journal/jap/118/20?ver=pdfcov

Published by the AIP Publishing

\section{AlP | APL Photonics}

APL Photonics is pleased to announce Benjamin Eggleton as its Editor-in-Chief

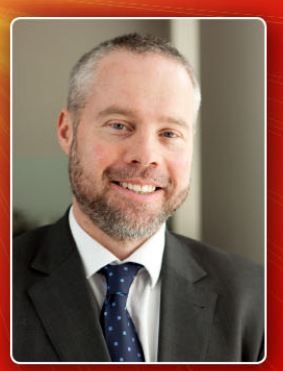




\title{
Magnetic characteristics of a high-layer-number NiFe/FeMn multilayer
}

\author{
G. W. Paterson, ${ }^{1, a)}$ F. J. T. Gonçalves, ${ }^{1}$ S. McFadzean, ${ }^{1}$ S. O'Reilly, ${ }^{2}$ R. Bowman, ${ }^{2}$ \\ and R. L. Stamps ${ }^{1}$ \\ ${ }^{1}$ SUPA, School of Physics and Astronomy, University of Glasgow, Glasgow G12 8QQ, United Kingdom \\ ${ }^{2}$ ANSIN, Department of Physics and Astronomy, Queen's University Belfast, Belfast BT7 INN, United Kingdom
}

(Received 13 August 2015; accepted 4 November 2015; published online 24 November 2015)

\begin{abstract}
We report the static and dynamic magnetic characteristics of a high-layer-number $\mathrm{NiFe} / \mathrm{FeMn}$ multilayer test structure with potential applications in broadband absorber and filter devices. To allow fine control over the absorption linewidths and to understand the mechanisms governing the resonances in a tailored structure similar to that expected to be used in real world applications, the multilayer was intentionally designed to have layer thickness and interface roughness variations. Magnetometry measurements show that the sample has complex hysteresis loops with features consistent with single ferromagnetic film reversals. Characterisation by transmission electron microscopy allows us to correlate the magnetic properties with structural features, including the film widths and interface roughnesses. Analysis of resonance frequencies from broadband ferromagnetic resonance measurements as a function of field magnitude and orientation provide values of the local exchange bias, rotatable anisotropy, and uniaxial anisotropy fields for specific layers in the stack and explain the observed mode softening. The linewidths of the multilayer are adjustable around the bias field, approaching twice that seen at larger fields, allowing control over the bandwidth of devices formed from the structure. (C) 2015 AIP Publishing LLC.
\end{abstract}

[http://dx.doi.org/10.1063/1.4936199]

\section{INTRODUCTION}

Exchange bias is often thought of as an effective unidirectional anisotropy that acts on a ferromagnet (FM) in contact with an antiferromagnet (AFM). ${ }^{1,2}$ Microwave frequency response is important for a number of device applications and has been explored within the context of exchange bias. ${ }^{3-7}$ The exchange bias of FM layers within a composite FM/AFM multilayer can be controlled by the FM layer thickness. Tailoring these thicknesses within a multilayer can give rise to a range in the absorption frequencies and has applications in broadband microwave shielding. ${ }^{8}$

The total effective field acting on an FM film affects the associated ferromagnetic resonance (FMR), and can create sizeable shifts in the resonance frequency. ${ }^{9,10}$ FMR has been used to study exchange bias as an effective bulk field in a number of different systems. ${ }^{11-18}$ Standing spin waves have also been used to study exchange bias as an interface pinning effect. $^{19}$

In this work, we investigate primarily using FMR the magnetic properties of a $\mathrm{Ni}_{0.8} \mathrm{Fe}_{0.2} / \mathrm{Fe}_{0.5} \mathrm{Mn}_{0.5}$ multilayer test structure. Structural and chemical analyses show layer thickness and interface roughness variations across the sample and we are able to correlate magnetic features with specific layers, including the influence of layer position within the stack. Through careful analysis, we determine the distribution of exchange biases and uniaxial and rotatable anisotropies within the multilayer. Due to structural imperfections, the resonances of the stack are spread over a wide range of frequencies. The resonance widths increase dramatically at the bias field as the modes soften due to changes in magnetic

\footnotetext{
${ }^{\text {a)} E l e c t r o n i c ~ m a i l: ~ g a r y . p a t e r s o n @ g l a s g o w . a c . u k ~}$
}

configuration. These properties show the system to be a promising one for using in broadband filters in which the bandwidth and absorption frequency may be tuned.

\section{SAMPLE GROWTH, STRUCTURE, AND MAGNETISATION}

The sample under study is a NiFe/FeMn multilayer test structure produced in a custom system built by Queens University using methods based on industrial standard processes. In the process, alternate films were sputtered in a $5 \mu$ bar Argon atmosphere onto an oxidised Si substrate and the resulting stack was capped with $5 \mathrm{~nm}$ of Ta to protect it from oxidation. The sample has eleven FM films and begins and ends with $\mathrm{NiFe}$ layers, giving twenty exchange biased interfaces. Post-deposition, the sample was annealed in a $0.2 \mathrm{~T}$ field at $280^{\circ} \mathrm{C}$ for $1 \mathrm{~h}$ to define an easy axis.

Transmission electron microscopy (TEM) and scanning TEM (STEM) with an elemental analysis in a JEOL ARM200F was used on a focussed ion beam prepared ${ }^{20}$ crosssection of the sample to determine the film thicknesses, interface quality, and distribution of chemical species. These and all other measurements reported were performed at room temperature. The film thicknesses can be seen in the angular dark field image of Figure 1(a). Excluding the outermost films, the $\mathrm{NiFe}$ film thicknesses within the stack vary slightly but are generally similar, with an average and standard deviation of $16.5 \pm 0.5 \mathrm{~nm}$. Of the outermost NiFe films, the first is close to average thickness, but the last is significantly thinner than all the other NiFe films. The small variations in the thickness of each layer cause the interface roughness to gradually build up with layer number, reaching a maximum deviation of approximately $\pm 2 \mathrm{~nm}$ at the Ta interface. The difference in roughness 

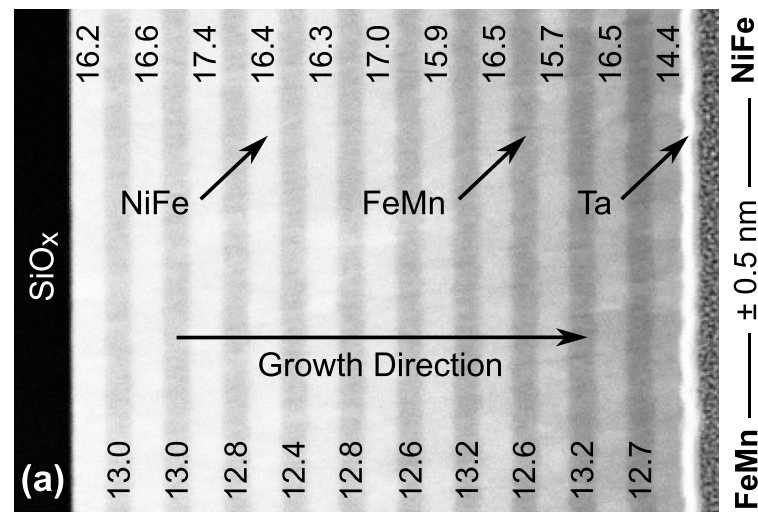

(b)
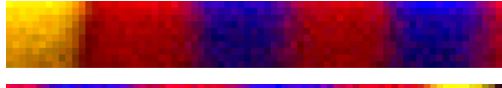

(c)

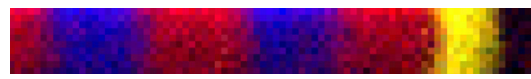

Ni Mn $\bigcirc$

$\mathrm{Ni} \mathrm{Mn} \mathrm{T⿹}$

FIG. 1. (a) Annular dark field scanning TEM (STEM) image showing layer structure and film widths. Energy filtered maps of areas at the (b) bottom and (c) top edges of the stack, show the indicated elemental distributions. Note that the elements coloured "yellow" are different for each map.

can also be seen in Figs. 1(b) and 1(c), which show maps of the distributions of the elements $\mathrm{Ni}$ (red), $\mathrm{Mn}$ (blue), $\mathrm{O}$, and $\mathrm{Ta}$ (yellow), at the bottom and top of the stack, respectively.

Consequences of the thickness distributions are apparent in magnetization loops determined by vibrating sample magnetometry (VSM), examples of which are shown in Fig. 2, where the magnetisation $\mathrm{M}$ is plotted as a function of field $\mathrm{H}$. The loop structure is suggestive of five discrete steps in magnetisation. The four "loops" labelled 1 to 4 each have a change in magnetisation of approximately $1 / 11$ of the total change in saturation magnetisation of the sample and are consistent with single film reversals. The fifth "loop" is much larger than the others and must result from material across several layers.

The small steps in magnetisation are separated by fields of $2-3 \mathrm{mT}$ and in an otherwise uniform sample would correspond to sequential reversal of progressively thinner FM films at larger fields. To understand this, suppose that the only anisotropy is unidirectional so that the effective exchange energy associated with each interface has

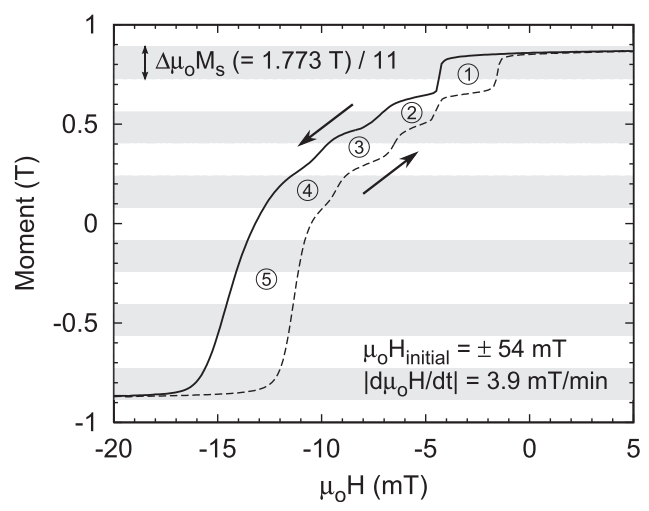

FIG. 2. Magnetisation data showing five discrete changes in value, measured in a PMC MicroMag 3900 VSM. The magnetic induction was converted in to magnetisation using the sample area and the total measured FM thickness. The large arrows indicate the applied field sweep direction. magnitude $E_{\text {int }}$. At the outermost films, which have only one exchange biased interface, reversal will occur when the applied field $H=E_{\text {int }} / M t$, where $M$ and $t$ are the NiFe film magnetisation and thickness. The other $\mathrm{NiFe}$ films will reverse with $H=2 E_{\text {int }} / M t$. We would therefore expect that the thickest outermost NiFe film should be the first to reverse. This film is adjacent to the substrate and we note that the minor loop associated with this reversal is also the one most clearly defined. The other loops are less well defined, which may indicate non-uniform reversal processes, possibly associated with an $E_{\text {int }} / t$ that varies laterally along the multilayer. We also note that the spread in biases is much larger than that expected from the range of film thicknesses alone, indicating $E_{\text {int }} / t$ also varies longitudinally across the deposition direction. One possible reason for this is the increasing interface roughness with growth, which can decrease the unidirectional interface coupling due to an increased number of uncompensated spins at the AFM/FM interface being free to rotate with the FM magnetisation. ${ }^{21}$ If this is the case, one would expect the presence of rotatable anisotropy (RA) in our sample. RA is not directly observable in magnetostatic measurement; however, as discussed later, our FMR data does reveal RA to be present in our sample.

\section{RESONANCE DISTRIBUTIONS AND WIDTHS}

To understand how the microwave frequency properties of the multilayer depend on the distribution of $E_{\text {int }} / t$, FMR measurements were made using a Rhode \& Schwarz ZVA40 vector network analyser at power of $5 \mathrm{dBm}$ with a microstrip waveguide microwave circuit. The resonance frequency of a single thin film is determined by local effective fields that describe torques governing the precession of the magnetisation. For FMR in thin films, the dominant contribution to these effective fields is the demagnetisation field, which is proportional to the magnetization and $E_{\text {int }} / M t$, the field associated with the unidirectional anisotropy. To assess the presence and magnitudes of the anisotropies in the sample, FMR measurements were performed at a number of sample angles measured relative to a static external field applied perpendicular to the magnetic component of the microwave field. We define the angle with the field applied parallel to the annealing field (used to set the direction of the unidirectional anisotropy) as $90^{\circ}$. At each angle, the S21 scattering parameter (the forward power transmission coefficient) was recorded for a range of frequencies over a range of fields. The data were then normalised and processed to reduce background variations as discussed in the supplementary material. ${ }^{22}$ The field was swept over an approximately symmetric range of values and always from positive to negative.

The FMR response of the 11-NiFe-layer stack is presented as frequency vs. field, with the field applied along the bias direction, in Figure 3(a). The data acquired at different applied field angles can be seen in the supplementary material. ${ }^{22}$ In Figures 3(b) and 3(c), the same data from a NiFe/ $\mathrm{FeMn}$ bilayer and a $\mathrm{NiFe} / \mathrm{FeMn} / \mathrm{NiFe} / \mathrm{FeMn}$ are shown for comparison. Here, the increase in exchange bias of FM layers with two rather than one interface bounded by AFM layers discussed above is clearly demonstrated. Unlike in stacks of 


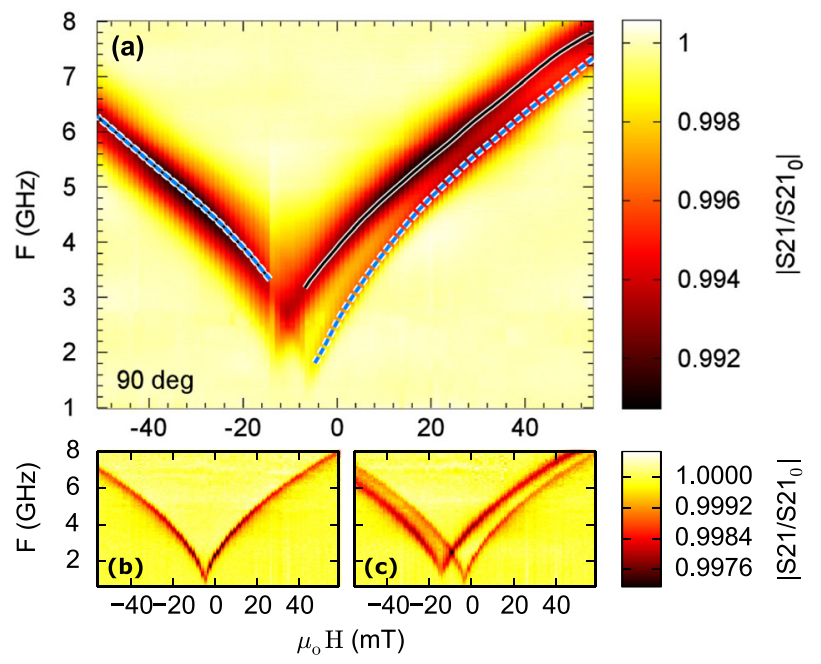

FIG. 3. (a) FMR spectra from the 11-NiFe-layer sample with the applied field aligned parallel to the bias direction. (b) and (c) are the equivalent data from multilayer samples nominally comprised by $\mathrm{NiFe}(20 \mathrm{~nm}) / \mathrm{FeMn}(10 \mathrm{~nm})$ and $[\mathrm{NiFe}(20 \mathrm{~nm}) / \mathrm{FeMn}(10 \mathrm{~nm})] \times 2$, respectively, each capped by $5 \mathrm{~nm}$ of Ta The lines in (a) are frequencies extracted by fitting Lorentzian functions to the high-(solid) and low-(dashed) frequency resonances.

fewer layers, which are more commonly studied, the individual resonances of each $\mathrm{NiFe}$ layer within the stack cannot be resolved. Instead, two overlapping resonances of different widths are observed, each much broader than that of a bi- or quad-layer samples. Although the inability to resolve the individual resonances limits the detail in which the response of the 11-NiFe-layer stack can be analysed, it is still important to study such stacks as they more closely replicate the structure that may be used in a real device. Additionally, even though there is evidence of our sample being imperfect, with, for example, interface couplings and roughnesses which vary across the sample, a degree of non-uniformity may in fact be beneficial in absorbers and filters where a broad but uniform response is desired. Consequently, the remainder of this work will focus exclusively on the 11-NiFe-layer sample.

Both resonances of the 11-NiFe-layer stack have a nonzero frequency minimum which is an indicator of the possible presence of rotational anisotropy. Evidence for uniaxial anisotropy can be observed by plotting the angular data in a polar form as shown in Figure 4. The data as a function of field at different applied field angles can be seen in the supplementary material. ${ }^{22}$ The oval is displaced relative to the plot centre because of the unidirectional anisotropy. The general shape of the curve is slightly oval, with a major axis along approximately $135^{\circ}-315^{\circ}$, indicating uniaxial anisotropy is present and weak in strength.

The FMR results correlate well with the loops observed from the VSM data. Of particular note is the sharp drop in the resonance amplitude just beyond the minimum of the lowfrequency resonance at around $-6 \mathrm{mT}$. This can be seen more clearly in Figure 5, in which we plot the field derivative of the FMR data to increase the contrast, and overlay the magnetisation hysteresis loop. Such a feature can occur in the resonance of a single film when the net magnetisation is close to zero, just as the moments flip, and corresponds to vanishing restoring torques. The low-frequency resonance dip occurs at a

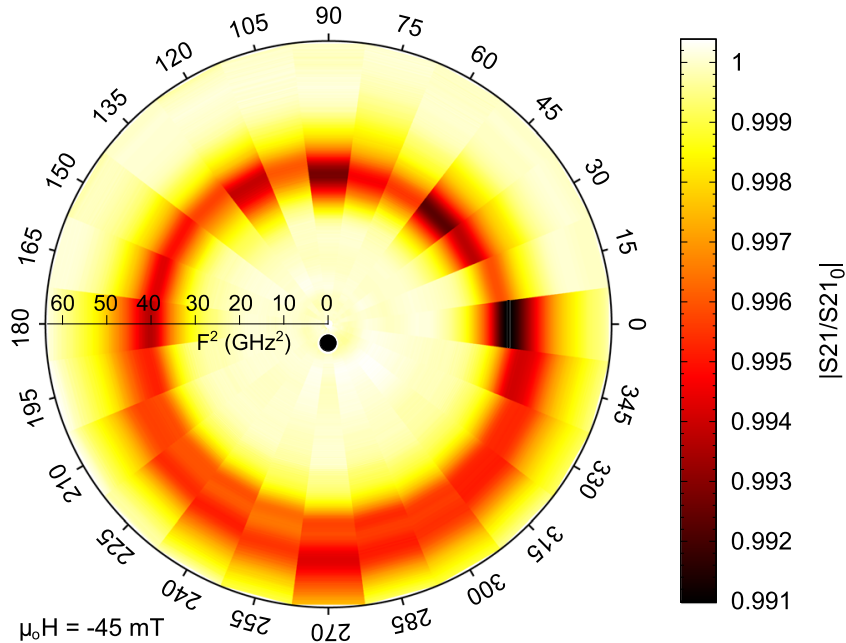

FIG. 4. Polar plot of the FMR response at a fixed large negative applied field, showing from the position and shape of the signal that uniaxial and unidirectional anisotropy are present in the sample, respectively. The symbol is the centroid of a single minimum at each angle.

similar field as the reversal of loop 1, as indicated in the figure, so we can attribute this resonance to the material responsible for that change in magnetisation. Thus, we conclude the low-frequency resonance results from the NiFe film adjacent to the substrate. A similar dip of a commensurately larger amplitude is present for the high-frequency resonance at approximately $-13 \mathrm{mT}$, at around which field loop 5 reverses. However, here the resonance is not from a single FM film, but results from nearly degenerate resonances of material across several films within the bulk of the stack.

While only two clear resonances have been identified here, there are hints of multiple overlapping resonances in the raw data. An example of this can be seen in the faint resonance intersecting the $y$-axis of Figure 5 at around $5.2 \mathrm{GHz}$. When undergoing resonant precession, the films are coupled to one another via dipolar fields, resulting in collective resonance modes. ${ }^{23,24}$ Of these collective excitations, only those with a net oscillating magnetic moment can be observed in FMR. Interestingly, a detailed analysis of FMR profile in which multiple overlapping Lorentzians are used to model the

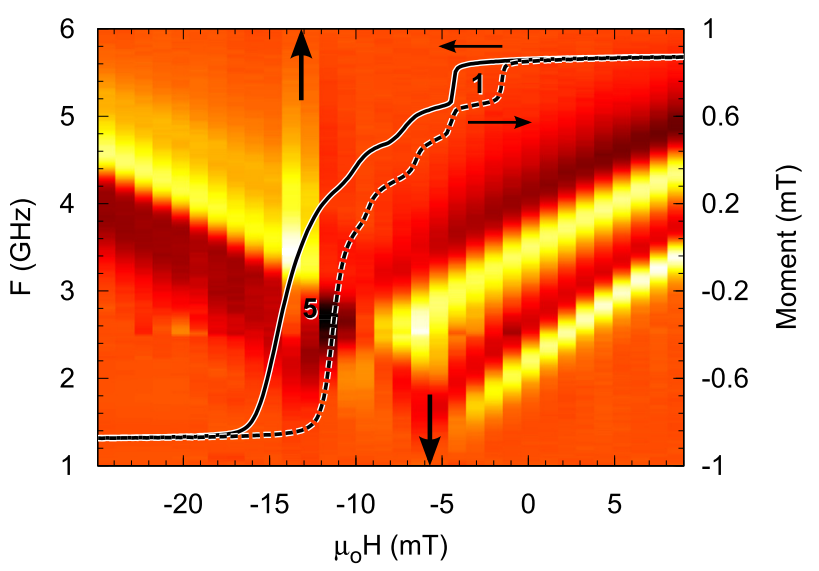

FIG. 5. Field derivative of the FMR data with overlaid VSM data at the bias angle. The vertical arrows indicate the position of the dips in FMR amplitude. 
linewidths is able to reproduce the observed peak profiles. However, as one might expect from a multilayer designed for use in broadband shielding, the resonances are not sufficiently well resolved to allow a thorough analysis.

\section{EXCHANGE BIAS AND ANISOTROPIES}

To quantify the anisotropies in the sample we analyse the FMR data by first determining the resonance frequencies and widths by fitting a Lorentzian function to each of the two resonances at every field value. The peak frequencies determined in this way are plotted as lines in Figure 3. The data at different applied field angles can be seen in the supplementary material. ${ }^{22}$ For field values where separate resonances could not be resolved, only one Lorentzian was used to determine a single peak frequency. Our treatment amounts to an implicit averaging over nearly degenerate excitations, although information is contained in the linewidth and peak profile.

Next, a Kittel-like equation is fitted to each of the two resonances at every angle of applied field, before assessing the angular dependence of the fitted parameters. We begin with (1), in which $M_{\text {eff }}$ is the effective magnetisation and $H_{a}$ $\& H_{a^{\prime}}$ represent anisotropy of any form

$$
f^{2}=\left(\frac{\gamma}{2 \pi}\right)^{2}\left(\left|H-H_{b}\right|+H_{a}\right)\left(\left|H-H_{b}\right|+H_{a^{\prime}}+M_{e f f}\right) .
$$

In our data, the anisotropy is small [Fig. 4] and the measurements are made in the low field range where the dominant contribution to $f^{2}$ is from $M_{e f f}$, as demonstrated by the linearity of $f^{2}(H) .{ }^{22}$ Consequently, we are unable to fit all parameters within the uncertainties of the measurements and, therefore, we make the assumption $\left|M_{e f f}\right| /|| H-H_{b}\left|+H_{a^{\prime}}\right| \gg 1$, which leads to the approximation used for the fittings

$$
f^{2} \approx\left(\frac{\gamma}{2 \pi}\right)^{2} M_{e f f}\left(\left|H-H_{b}\right|+H_{a}\right)
$$

The fits of (2) were performed using a LevenbergMarquart algorithm to data at applied fields larger than $\pm 15 \mathrm{mT}$ so that the magnetisation was saturated along the applied field direction [see Fig. 5]. The fits were weighted using the half width half maximum (HWHM) of the resonance and common effective magnetisations were used for each resonance across all data. All other parameters were allowed to vary independently of each other and of angle so that specific forms of anisotropy need not be presupposed.

The two effective magnetisation values extracted by the fit are shown in Table I. The $M_{\text {eff }}$ values are similar to one another and to the bulk permalloy saturation magnetisation of around $1060 \mathrm{mT}^{25}$ The somewhat higher values in the dynamic measurement reported here than the static one [Fig. 2] indicates some fraction of the total ferromagnetic volume is of lower moment. This is consistent with the effects of surface roughness and is also a source of linewidth broadening.

The angular dependence of the exchange bias and anisotropy is shown in Figure 6. The exchange bias of the material giving rise to both resonances is well explained by a
TABLE I. Summary of the parameters extracted from the FMR data.

\begin{tabular}{lccccc}
\hline \hline & High F & $\sigma$ & Low F & $\sigma$ & Unit \\
\hline$M_{e f f}$ & 1074.4 & 2.1 & 1090.8 & 1.4 & $\mathrm{mT}$ \\
$\mu_{o} H_{b}^{o}$ & -10.82 & 0.21 & -5.56 & 0.13 & $\mathrm{mT}$ \\
$\theta_{b}$ & 92.1 & 1.0 & 95.3 & 1.3 & $\mathrm{deg}$ \\
$\mu_{o} H_{r}$ & 5.16 & 0.27 & 0.02 & 0.16 & $\mathrm{mT}$ \\
$\mu_{o} K_{u}$ & 2.59 & 0.44 & 3.51 & 0.26 & $\mathrm{mT}$ \\
$\theta_{u}$ & 142.9 & 4.9 & 141.7 & 2.0 & $\mathrm{deg}$ \\
\hline \hline
\end{tabular}

cosine function (3) fitted to the data [Fig. 6(a)], in which $\theta_{b}$ is the bias angle. The same technique as used for the Kittel fit was used here and gave coefficients of determination, $\mathrm{r}^{2}$, values of around 0.99 . The extracted parameter values and their standard deviations are listed in Table I. The magnitude of the exchange bias, $H_{b}^{o}$, of the high frequency resonance is a little under twice that of the low frequency one, consistent with differences associated with the $H_{b}$ fields acting on the inner and outermost NiFe films, as discussed earlier

$$
H_{b}=H_{b}^{o} \cos \left(\theta-\theta_{b}\right) .
$$

Both anisotropy curves [Fig. 6(b)] show the two-fold symmetry of uniaxial anisotropy but the magnitudes and the offsets from zero field are markedly different. The constant offset in the high frequency curve confirms the presence of a rotatable anisotropy, where uncompensated spins at the AFM/ FM interface rotate with the FM magnetisation. ${ }^{26}$ The lines in Fig. 6(b) are fits to the data of (4), in which $H_{r}$ is the rotatable anisotropy term and the second term represents uniaxial anisotropy of strength $K_{u}$ with a hard axis at angle $\theta_{u}$. The fit to the high frequency resonance $\left(\mathrm{r}^{2}=0.63\right)$ is worse than that for the low frequency resonance $\left(r^{2}=0.90\right)$, reflecting the nature of modelling multiple overlapping resonances as a single resonance. The parameters extracted by these fittings are also listed in Table I. The uniaxial anisotropy is aligned at the same angle in both resonances and is stronger in the low frequency resonance which, unlike the high frequency resonance, has no measurable rotatable anisotropy field

$$
H_{a}=H_{r}+K_{u} \cos ^{2}\left(\theta-\theta_{u}\right) .
$$

We believe these differences are the result of differences in interface coupling due to changes in interface roughness across the stack. Higher interface roughness can increase the RA and decrease the other anisotropies because the
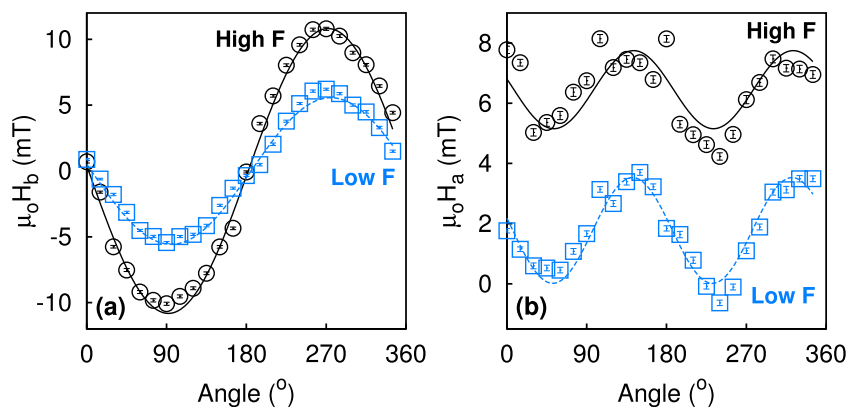

FIG. 6. Fits (lines) to the extracted (a) $H_{b}$ and (b) $H_{a}$ angular data (symbols) for both resonances. The error bars are the standard deviations. 


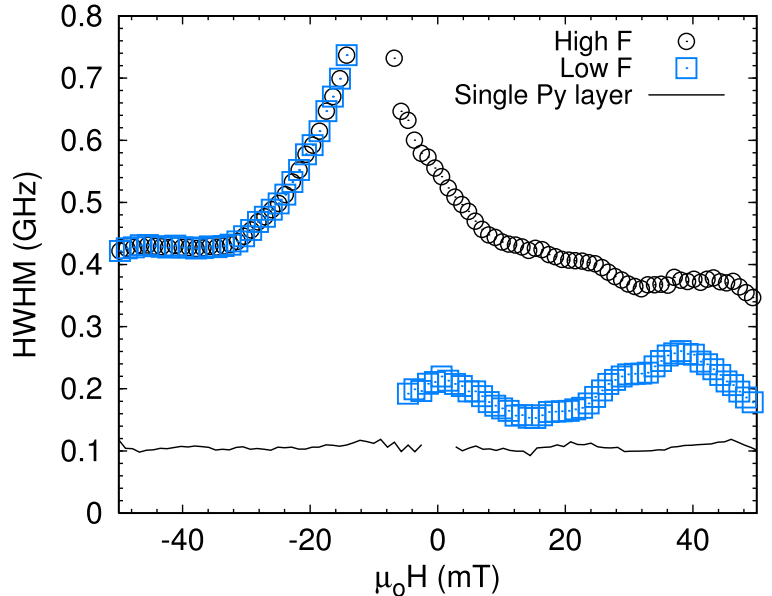

FIG. 7. Comparison of the linewidths in the multilayer sample along the bias direction and a sample comprised by a single $30 \mathrm{~nm}$ thick $80 \%$ Py film.

interfacial area with grains of rotatable magnetisation increases and, therefore, a smaller area remains to contribute to the uniaxial and unidirectional anisotropies. This provides further support for the low frequency resonance resulting from the single NiFe film adjacent to the substrate, which has a significantly smoother interface than the top NiFe film. Thus, through careful analysis of TEM, VSM, and FMR data we are able to identify and analyse the properties of an individual layer within the stack.

Finally, the linewidths obtained from the multilayer sample are compared with a reference sample comprised by a single Py film in Figure 7. The multilayer sample resonance widths are up to $4 \times$ that of the single film sample at high fields and diverge at the bias field. The divergence is to be expected since at this field magnetizations of individual films will reverse, changing the magnetic configuration and leading to mode softenings. This feature is a consequence of the near degenerate resonances of layers with a distribution of biases and anisotropies associated with the non-uniform film thicknesses and interface couplings resulting from the structural imperfections, and could be exploited in devices to allow bandwidth tuning.

\section{CONCLUSIONS}

We have studied in detail the static and dynamic magnetic properties an imperfect exchange biased multilayer $\mathrm{NiFe} / \mathrm{FeMn}$ test sample and found good agreement between them. The sample is characterised by a distribution of biases and rotatable \& uniaxial anisotropies. These properties, the result of structural imperfections, lead to greatly broadened resonance linewidths which diverge at the bias field, demonstrating the $\mathrm{NiFe} / \mathrm{FeMn}$ system as a promising one for use in broadband filters in which the bandwidth and absorption frequency may be tuned.

\section{ACKNOWLEDGMENTS}

This work was supported by EPSRC Bridging the Gap and the University of Glasgow. We acknowledge the assistance of Dr. T. D. Drysdale in developing the FMR measurement system at Glasgow.

${ }^{1}$ W. H. Meiklejohn and C. P. Bean, Phys. Rev. 102, 1413 (1956).

${ }^{2}$ J. Nogues and I. K. Schuller, J. Magn. Magn. Mater. 192, 203 (1999).

${ }^{3}$ S. Li, Z. Huang, J.-G. Duh, and M. Yamaguchi, Appl. Phys. Lett. 92, 092501 (2008).

${ }^{4}$ G. Srinivasan, A. S. Tatarenko, and M. I. Bichurin, Electron. Lett. 41, 596 (2005).

${ }^{5}$ M. I. Bichurin, D. Viehland, and G. Srinivasa, J. Electroceram. 19, 243 (2007).

${ }^{6}$ C. S. Tsai and J. Su, Appl. Phys. Lett. 74, 2079 (1999).

${ }^{7}$ C. S. Tsai and J. Su, IEEE Trans. Magn. 35, 3178 (1999).

${ }^{8}$ N. N. Phuoc, F. Xu, and C. K. Ong, Appl. Phys. Lett. 94, 092505 (2009).

${ }^{9}$ R. Stamps, R. E. Camley, and R. J. Hicken, Phys. Rev. B 54, 4159 (1996).

${ }^{10}$ W. Stoecklein, S. S. P. Parkin, and J. C. Scott, Phys. Rev. B. 38, 6847 (1988).

${ }^{11}$ S. Queste, S. Dubourg, O. Acher, K. U. Barholz, and R. Mattheis, J. Appl. Phys. 95, 6873 (2004).

${ }^{12}$ B. K. Kuanr, R. E. Camley, and Z. Celinsky, J. Appl. Phys. 93, 7723 (2003).

${ }^{13}$ J. Yang, S. Cardoso, P. P. Freitas, T. Devolder, and M. Ruehrig, J. Appl. Phys. 109, 07D704 (2011).

${ }^{14}$ J. Yang, S. Cardoso, P. P. Freitas, T. Devolder, and M. Ruehrig, Appl. Phys. Lett. 97, 132502 (2010).

${ }^{15}$ H. Xi, J. Rantschler, S. Mao, M. T. Kief, and R. M. White, J. Phys. D: Appl. Phys. 36, 1464 (2003).

${ }^{16}$ D. Spenato, S. P. Pogossian, D. T. Dekadjevi, and J. Ben Youssef, J. Phys. D: Appl. Phys. 40, 3306 (2007).

${ }^{17}$ J. Geshev, L. G. Pereira, J. E. Schmidt, L. C. Nagamine, E. B. Saitovitch, and F. Pelegrini, Phys. Rev. B 67, 132401 (2003).

${ }^{18}$ M. J. Pechan, D. Bennett, N. Teng, C. Leighton, J. Nogus, and I. K. Schuller, Phys. Rev. B 65, 064410 (2002).

${ }^{19}$ R. Magaraggia, K. Kennewell, M. Kostylev, R. L. Stamps, M. Ali, D. Greig, B. J. Hickey, and C. H. Marrows, Phys. Rev. B 83, 054405 (2011).

${ }^{20}$ J. Mayer, L. A. Giannuzzi, T. Kamino, and J. Michael, MRS Bull. 32, 400 (2007).

${ }^{21}$ M. Pakala, Y. Huai, G. Anderson, and L. Miloslavsky, J. Appl. Phys. 87, 6653 (2000)

${ }^{22}$ See supplementary material at http://dx.doi.org/10.1063/1.4936199 for an example of FMR data processing, videos of FMR dependence on applied field angle and magnitude, and fits of the Kittel equation to the data.

${ }^{23}$ B. Hillebrands, Phys. Rev. B 41, 530-540 (1990).

${ }^{24}$ R. E. Camley and R. L. Stamps, J. Phys.: Condensed Matter 5, 3727 (1993).

${ }^{25}$ J. M. D. Coey, Magnetism and Magnetic Materials (Cambridge University Press, 2009), p. 343.

${ }^{26}$ R. Dutra, D. E. Gonzalez-Chavez, T. L. Marcondes, A. M. H. de Andrade, J. Geshev, and R. L. Sommer, J. Magn. Magn. Mater. 346, 1 (2013). 